\title{
Association of HLA Alleles with Primary Sjögren Syndrome in the South Tunisian Population
}

\author{
Aida Charfi ${ }^{a}$ Nadia Mahfoudh ${ }^{a}$ Arwa Kamoun $^{a}$ Feten Frikha $^{b}$ \\ Chifa Dammak $^{b}$ Lilia Gaddour ${ }^{a}$ Feiza Hakim ${ }^{a}$ Leila Maalej ${ }^{a}$ Bakhta Mallek ${ }^{a}$ \\ Ines Kammoun ${ }^{\mathrm{a}}$ Zouhir Bahloul ${ }^{\mathrm{b}}$ Hafedh Makni ${ }^{\mathrm{a}}$ \\ a Department of Histocompatibility, Hedi Chaker University Hospital, Sfax, Tunisia; \\ ${ }^{b}$ Department of Internal Medicine, Hedi Chaker University Hospital, Sfax, Tunisia
}

\section{Significance of the Study}

- HLA-DR and DQ alleles represent the most common associations reported in primary Sjögren syndrome (pSS).

- In this study, besides HLA-DRB1*03, we found HLA-B15 association with pSS in a cohort of 44 South Tunisian patients.

- Microsatellite analysis supported the hypothesis of the existence of a susceptibility gene to pSS located in the HLA class I and III region.

\section{Keywords}

Primary Sjögren syndrome · Human leukocyte antigen ·

Polymorphism · Microsatellite · Haplotype

\begin{abstract}
Objective: In order to investigate human leukocyte antigen (HLA) genes predisposing to primary Sjögren syndrome (pSS), we conducted an association study using HLA loci (A, $B$, and DRB1) and 9 polymorphic microsatellite markers spanning the HLA region in pSS patients as compared to healthy individuals. Subjects and Methods: Forty-four patients fitting the European criteria of pSS and 123 healthy controls were analyzed for their HLA class I and class II alleles. HLA class I typing was performed using a standard microlym-
\end{abstract}

\section{KARGER}

E-Mail karger@karger.com www.karger.com/mpp (c) 2019 The Author(s) Published by S. Karger AG, Basel

Karger Open access

This is an Open Access article licensed under the Creative Commons Attribution-NonCommercial-4.0 International License (CC BY-NC) (http://www.karger.com/Services/OpenAccessLicense), applicable to the online version of the article only. Usage and distribution for commercial purposes requires written permission. phocytotoxicity method followed by PCR-SSP. HLA-DRB1 genotyping was performed using PCR-SSP. We studied the polymorphism of 9 microsatellite markers for both groups. Microsatellite genotyping was performed using the PCR fluorescent technique. Results: We observed a positive association between HLA-B15 and pSS in the Tunisian population ( $p=0.004$, OR 7.57). The comparison of the frequencies of DRB1 alleles in pSS patients and controls confirmed the association of the DRB $1 * 03$ allele with $\mathrm{pSS}(p=0.02$, OR 2.36). On the other hand, the association study of microsatellite markers showed that the a9 allele of D6S265 marker and the a20 of C1.2.C were found to be positively associated with pSS as compared to controls ( $p=0.0003$, OR 10.29 , and $p=0.001$, OR 4.79, respectively). Using the "Haplo.stats" software analysis, we found that the most associated region was located

Aida Charf

Department of Histocompatibility, Hedi Chaker Hospital

Route El Ain

Sfax 3000 (Tunisia)

E-Mail aydack@gmail.com 
in the HLA class I region and limited by HLA-A and D6S265 loci $(p=0.00056)$. Conclusion: The results of this study support the hypothesis of the existence of a susceptibility gene for pSS located in the HLA class I and III regions.

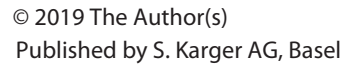

\section{Introduction}

Sjögren syndrome (SS) is a common, slowly progressive autoimmune disease (AID) characterized by chronic infiltration in the exocrine glands, particularly affecting the salivary and lacrimal glands, which causes conjunctival and oral dryness [1]. SS is called primitive when it manifests in the absence of any other AID such as rheumatoid arthritis (RA), systemic lupus erythematosus (SLE), scleroderma, and myositis [2]. In addition to the glandular manifestations, extra-glandular manifestations affecting the skin, joints, muscles, nervous system, lungs, kidneys, liver, and hematological involvement may occur [1]. Primary SS (pSS) is one of the most common connective tissue diseases with an estimated prevalence of $0.2-$ $3.39 \%$ in the general population. This pathology preferentially affects women (sex ratio $=9.15$ ) between the fourth and fifth decades of life.

SS is a multifactorial disease related to genetic, hormonal, and environmental factors. However, while much progress has been made in recent decades, the pathophysiology of pSS remains unclear. Susceptibility to developing SS has been strongly associated with human leukocyte antigen (HLA) class II genes, particularly some HLA-DR and DQ alleles. Thus, the search for other genes in and/ or outside the HLA system is essential. Several approaches have been used, such as the Genetic Linkage analysis, positional candidate gene analysis using microsatellite markers, and more recently the genome-wide association studies conducted on a large series of patients and controls with SNPs (single nucleotide polymorphism) markers $[3,4]$.

Only one previous microsatellite association study has been performed in the Tunisian population [5], while no association study between pSS and HLA polymorphism (A, B, and DR) has been conducted. Here, we report a case-control study of HLA-A, B, DR alleles and nine microsatellite markers distributed in the HLA region on a sample of 44 patients with pSS and 123 healthy controls from the South Tunisian population in order to identify other susceptibility loci on the short arm of chromosome 6.

HLA and Primary Sjögren Syndrome
Table 1. Distribution of HLA markers in South Tunisian patients with pSS and controls

\begin{tabular}{lll}
\hline & Marker & $p$ \\
\hline HLA-I & D6S276 & NS \\
& HLA-A & NS \\
& D6S265 & 0.0002 \\
& C3-2-11 & NS \\
HLA-III & HLA-B & 0.0002 \\
& MICA & NS \\
& C1_2_C & 0.04 \\
& TNFa & NS \\
HLA-II & TNFC & 0.03 \\
& D6S273 & NS \\
& HLA-DR & NS \\
& D6S291 & NS \\
\hline
\end{tabular}

$p$ values are from Pearson's test. HLA, human leukocyte antigen; pSS, primary Sjögren syndrome; NS, not significant.

\section{Subjects and Methods}

\section{Subjects}

Our case-control association study was focused on a representative sample of the South Tunisian population. The study included 44 patients with pSS and met the criteria established in 2002 by the American European Consensus Group (AECG) [2], clinically followed at the Internal Medicine Department of the Hedi Chaker University Hospital in Sfax between 2012 and 2015. Our control group was made up of 123 healthy unrelated subjects (72 women and 51 men, mean age $32 \pm 9.28$ years) from the south of Tunisia [6]. All patients and controls gave written informed consent for their participation in the study. This study was approved by our local ethics committee.

Methods

Data Collection

For each patient, we collected the following data: age, gender, clinical and para-clinical data, as well as biological and immunological data (anti-SSA and anti-SSB antibodies; Ab).

HLA Typing

We performed HLA class I typing (A and B) using a standard microlymphocytotoxicity method followed by PCR-SSP (One Lambda ${ }^{\circledR}$ ). HLA class II (DRB) genes were typed using a polymerase chain reaction-sequence specific primer (PCR-SSP) kit (One Lambda ${ }^{\circledR}$ ).

Microsatellite Markers

For our study we chose nine microsatellite markers that covered the entire HLA region: D6S276, D6S265, C3-2-11 (D6S2701), MICA, C1_2_C (D6S2800), TNFa, TNFc, D6S273, and D6S291 from telomeric to centromeric chromosome 6 . The alleles of the markers have been designated by the repetition number of the simple pattern. Genotyping of the microsatellites of the two groups was performed by a fluorescent PCR technique, with specific amplification primer pairs (Perkin-Elmer, Applied Biosystems ${ }^{\circledR}$ ) [7].

Med Princ Pract 2020;29:32-38

DOI: $10.1159 / 000501896$ 
Table 2. Distribution of HLA and microsatellites alleles according to biological signs in pSS

\begin{tabular}{llllll}
\hline & \multicolumn{2}{c}{ Anti-SSA Ab (+ vs. -$), \%$} & Anti-SSB Ab (+ vs. -$), \%$ & $p$ & OR (95\% CI) \\
\hline \multirow{2}{*}{ HLA-I } & a6 (D6S276) & - & 4.5 vs. 31.8 & 0.046 & $0.10(0.01-0.91)$ \\
& a16 (D6S276) & 40.7 vs. 5.9 & - & 0.015 & $11(1.26-95.49)$ \\
& a11 (D6S265) & 18.5 vs. 47.1 & - & 0.043 & $0.25(0.06-0.99)$ \\
& B15 & 25.9 vs. 0 & - & 0.032 & - \\
HLA-III & B15 & - & 31.8 vs. 0 & 0.009 & - \\
HLA-II & DRB1 ${ }^{*} 03$ & - & - & 0.015 & $11(1.26-95.49)$ \\
& DRB1 04 & 40.7 vs. 5.9 & 63.6 vs. 22.7 & 0.006 & $5.95(1.58-22.33)$ \\
\end{tabular}

$p$ values are from Pearson's test. HLA, human leukocyte antigen; pSS, primary Sjögren syndrome.

Statistical Analysis

The statistical analysis was performed using the software SPSS20, SHeSis, and Haplo.stats (R language 3.3.2). $\chi^{2}$ and nominal $p$ value (Pearson's test) calculation were used to evaluate the association of HLA polymorphisms with pSS. Fisher's exact test for small sample sizes (lower than 5 units per cell) was applied to the $2 \times 2$ tables as appropriate. The risk of association was reported as an odds ratio (OR) with a confidence interval (CI) of $95 \%$. The significance threshold was set at $p<0.05$. A binary logistic regression test was used to assess the independence of different alleles associated with pSS.

\section{Results}

\section{Descriptive Study}

The 44 patients consisted of 39 women $(88.6 \%)$ and 5 men corresponding to a sex ratio of 0.12 (7.8 women for 1 man). The mean age of onset was 46.8 years (+/ -12.2 years). Forty patients (90.9\%) had anti-nuclear $\mathrm{Ab}, 27$ patients had anti-SSA Ab (61.4\%), and 22 had anti-SSB Ab (50\%).

\section{HLA/pSS Association Study}

The overall distribution of HLA-A antigens was not significantly different between patients and controls $(p=$ $0.06)$. For HLA-B antigens, the difference in antigen distribution between the two groups was significant $(p=$ 0.0002 ; Table 1). A positive association of HLA-B15 antigen was found in our patients compared to controls (15.91\% in pSS vs. $2.44 \%$ in controls; $p=0.004$, OR 7.57, 95\% CI 1.8-30.7). All subjects with HLA-B15 had antiSSA and anti-SSB Ab. This association was significant with $p$ values of 0.032 and 0.009 , respectively (Table 2 ). HLA-B8 was more frequent in patients compared to controls ( $25 \%$ in pSS vs. $13.01 \%$ in controls), but the difference was not significant $(p=0.06)$.

For HLA class II, a positive association of the HLADRB $1 * 03$ allele was observed in our patients as compared to controls (43.18\% in pSS vs. $24.39 \%$ in controls, $p=0.02$, OR 2.36, 95\% CI 1.14-4.86). The HLA-DRB1*01 allele was negatively associated with pSS compared to controls $(2.27 \%$ in pSS vs. $18.7 \%$ in controls, $p=0.01$, OR $0.1,95 \%$ CI $0.01-$ $0.77)$.The frequency of the $\mathrm{DRB} 1^{*} 04$ allele in patients $(27.27 \%)$ was close to that found in controls (30.08\%). However, HLA-DRB1*03, DRB1*04 heterozygosity was significantly more frequent in patients than in controls (13.64\% in pSS vs. $2.44 \%$ in controls, $p=0.004$, OR $6.3,95 \%$ CI 1.5-26.47). As for immunological signs, the DRB $1 * 04$ allele was associated with anti-SSA Ab $(p=0.015$, OR 11, 95\% CI 1.26-95.5) while the HLA-DRB1*03 allele was positively associated with the presence of anti-SSB Ab ( \pm antiSSA Ab; $p=0.006$, OR 5.95, 95\% CI 1.58-22.32; Table 2).

\section{Microsatellites/pSS Association Study}

The overall microsatellite association analysis with pSS showed a significant difference for the markers: D6S265, C1_2_C, and TNFc (Table 1). For microsatellite markers in the HLA class I region, only the D6S265 marker showed a positive association with pSS. The a9 allele was significantly more frequent in patients compared to controls $(20.45 \%$ in pSS vs. $2.44 \%$ in controls, $p=0.0003$, OR 10.29, 95\% CI 2.64-40.06). The binary logistic regression analysis showed that this association was independent of HLA-B15 (HLA-B15: $p=0.009$, OR 6.9; the a9 allele of D6S265: $p=0.001$, OR 9.6).

Comparing the group of patients with that of the controls, no association was found for the marker D6S276. However, the a16 allele of the D6S276 marker was associated with the presence of anti-SSA Ab (40.7\% in pSS vs. $5.9 \%$ in controls, $p=0.015$, OR $11,95 \%$ CI 1.26-95.49). In contrast, the a6 allele was negatively associated with the presence of anti-SSB Ab (4.5\% in pSS vs. $31.8 \%$ in controls, $p=0.046$, OR $0.10,95 \%$ CI $0.01-$ 0.91 ; Table 2).
Charfi et al. 


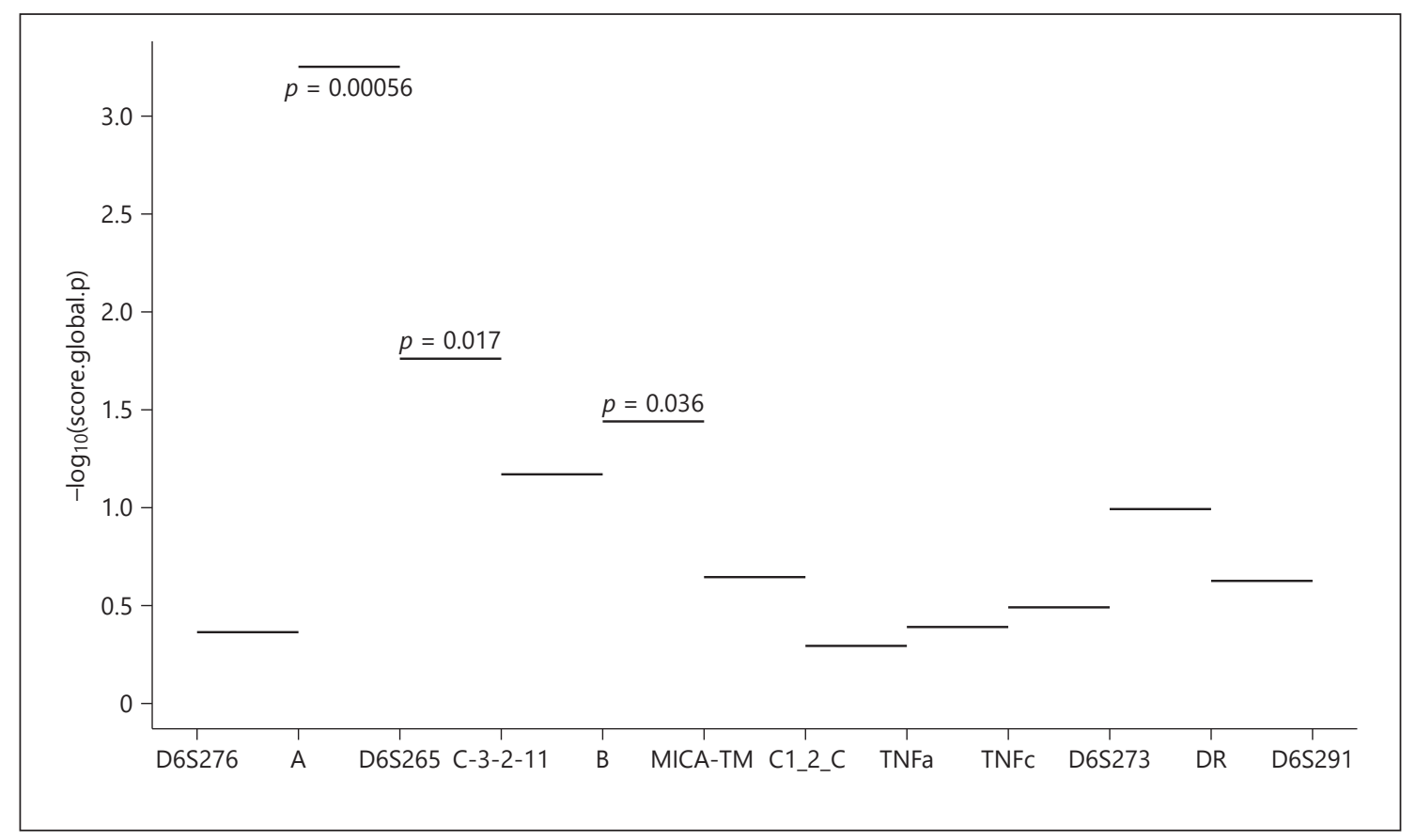

Fig. 1. Haplotype distribution for patients compared to controls by Haplo.stats software.

For microsatellite markers in the HLA class III region, the a20 allele of the C1_2_C marker was positively associated with pSS ( $25 \%$ in pSS vs. $6.5 \%$ in controls, $p=0.001$, OR 4.79, 95\% CI 1.7-12.8). The binary logistic regression analysis showed that the combination of the marker C1_2_C allele a20 with the pSS was independent of all other markers. The TNFc marker a 2 allele and the D6S273 marker a13 allele were negatively associated with pSS (36.36\% in pSS vs. $53.66 \%$ in controls, $p=0.05$, and $15.91 \%$ in pSS vs. $30.89 \%$ in controls; $p=0.05$, OR 0.42 , respectively). No allele of the MICA or TNFa markers were associated with the disease. The a 6 allele of the TNFa marker was associated with anti-SSA Ab $(p=0.015$, OR 11, 95\% CI 1.26-95.4; Table 2). No allele of the D6S291 marker was associated with the pSS in the HLA class II region.

\section{Haplotype-Association Analysis}

The study of haplotypes was carried out using Haplo. stats software. The most strongly associated region was localized in the HLA class I region and bounded by the A and B loci. A significant difference in haplotype distribution between patients or controls was found with three haplotype combinations: A-D6S265 ( $p=0.00056)$; D6S265-C3_2_1 $(p=0.017)$, and B-MICA $(p=0.036$; Fig. 1).

HLA and Primary Sjögren Syndrome

\section{Discussion}

SS is a common AID, known to be the second in terms of frequency after RA. However, few studies have focused on the Tunisian population, from which only one previous microsatellite association study has been reported [ 5 , 8]. Here, we report a case-control association study of the HLA-A, B, DR alleles and nine microsatellite markers distributed within the HLA region, on a sample of 44 patients with pSS and 123 healthy controls in South Tunisia. We have described for the first time an association between HLA-B15 and pSS in our population. As with most AID, an association of several HLA alleles with pSS have been described in different ethnic groups [9]. In the literature, the most HLA alleles involved in pSS susceptibility belong to the HLA class II region. Nevertheless, the HLA class I region has also been reported to be associated with pSS (Table 3). The first description of an association between HLA-B8 and SS in the Caucasian population was reported 40 years ago [10-12], but subsequent studies demonstrated that this association was due to linkage disequilibrium with HLA-DR3 [10]. In our study, HLA-B8 was more frequent in patients than in controls, but the difference was not significant. This could be explained by the relatively small number of patients $(n=44)$. Among HLA class I antigens, only HLA- 
Table 3. Summary of results from studies on the association of HLA class I and II with pSS

\begin{tabular}{|c|c|c|c|c|c|c|}
\hline $\begin{array}{l}\text { Population } \\
\text { (Reference) }\end{array}$ & Year & $\begin{array}{l}\text { Patients, } \\
n\end{array}$ & $\begin{array}{l}\text { Controls, } \\
n\end{array}$ & Associations found & $\begin{array}{l}\text { Pearson's } \\
p\end{array}$ & OR \\
\hline Caucasian (USA) [10] & 1978 & 19 & 96 & $\begin{array}{l}\text { HLA-B8 } \\
\text { HLA-DR3 }\end{array}$ & $\begin{array}{l}<0.0001 \\
<0.00001\end{array}$ & \\
\hline Caucasian [20] & 2003 & 149 & 222 & $\begin{array}{l}\text { HLA-DRB } 1^{*} 03 \\
\text { HLA-DQB1*02 }\end{array}$ & $\begin{array}{l}10^{-7} \\
0.0006\end{array}$ & $\begin{array}{l}3.8 \\
2.2\end{array}$ \\
\hline Colombian [21] & 2005 & 74 & 76 & $\begin{array}{l}\text { HLA-DRB }{ }^{*} 03: 01 \\
\text { HLA-DQB1*02:01 } \\
\text { Haplotype DRB1*03:01, } \\
\text { DQB1*02:01 }\end{array}$ & $\begin{array}{l}0.006 \\
0.02 \\
0.002\end{array}$ & $\begin{array}{l}1.9 \\
4.3 \\
3.6\end{array}$ \\
\hline $\begin{array}{l}\text { Meta-analysis: } \\
\text { Colombian [9] }\end{array}$ & 2012 & 1,166 & 6,470 & $\begin{array}{l}\text { HLA-DRB } 1 * 03: 01 \\
\text { HLA-DQA } 1 * 05: 01 \\
\text { HLA-DQB1*02:01 }\end{array}$ & $\begin{array}{c}<0.001 \\
<0.001 \\
0.01\end{array}$ & $\begin{array}{l}3,413 \\
2,276 \\
1,845\end{array}$ \\
\hline $\begin{array}{l}\text { Meta-analysis: } \\
\text { Chinese [22] }\end{array}$ & 2015 & 492 & 1,860 & HLA-DRB1*08:03 & 0.0002 & 3 \\
\hline Our series & 2016 & 44 & 123 & $\begin{array}{l}\text { HLA-B } 15 \\
\text { HLA-DRB } 1{ }^{*} 03 \\
\text { HLA-DRB }{ }^{*} 01 \\
\text { Heterozygotes DRB } 1{ }^{*} 03 \text { - } \\
\text { DRB }{ }^{*} 04\end{array}$ & $\begin{array}{l}0.004 \\
0.02 \\
0.01 \\
0.0047\end{array}$ & $\begin{array}{l}6.31 \\
0.1 \\
5.95\end{array}$ \\
\hline
\end{tabular}

HLA, human leukocyte antigen; pSS, primary Sjögren syndrome.

B15 was found to be positively associated with pSS in our series. This antigen has been associated particularly with the presence of anti-SSA and anti-SSB Ab. No previous review has reported the association of HLA-B15 antigen with pSS. However, the combination of HLA-B15 with other AID has been described by several research teams. This antigen has been associated with the peripheral form of ankylosing spondylitis in Colombians [13], with undifferentiated spondyloarthritis among Tunisians [14], and with the severe form of Behçet disease among Moroccans [15]. Other non-autoimmune pathologies (such as periodontitis) have been associated with HLAB15 [16]. The biological mechanism explaining the association of HLA class I molecules with pSS is not clear, but several hypotheses can be discussed and have been put forward.

An etiopathogenic role has been described for herpes viruses in the development of pSS [17]. A study by Rickinson and Moss [18] demonstrated the existence of a restricted representation of some antigenic EBV peptides "EBNA3" by HLA class I molecules, especially for HLAB15 (B62), B8, and A24. Molecular mimicry and pathogen co-capture may be involved in the pathogenesis of pSS [19].
Our study also implicates HLA class II alleles, especially the DRB $1{ }^{*} 03$ allele, in susceptibility to pSS. This association has been widely reported in the literature [9, 20,21 ] (Table 3). A meta-analysis of 1,166 pSS and 6,470 controls explained the association of the HLADRB1*03:01 allele with pSS by high affinity of HLA-DR3 for 24 antigenic peptides derived from SSA, SSB, $\alpha$-fodrin, and the muscarinic receptor (AChR-M3; the most likely antigenic targets during pSS) [9]. Similarly, the association of the DRB $1 * 08: 03$ allele in Chinese pSS patients was explained by the particularity of the antigen in cleft of this allele (tyrosine at position 47 and isoleucine or leucine at position 67 of the $\beta$-chain) [22].

Regarding the immunological profile of our patients, the anti-SSB and anti-SSA Ab were respectively associated with the DRB $1 * 03$ and DRB1*04 alleles. In Caucasians, anti-SSA Ab were associated with HLADRB $1 * 03: 01[20,21]$. A French study showed that simultaneous production of anti-SSA and anti-SSB Ab was associated with HLA-DRB1*03, while production of isolated anti-SSA was associated with DRB1*15 [20].

The HLA-DRB $1 * 01$ allele was negatively associated with the pSS in our series, a result which is consistent with a Hungarian study [23]. The protective role of the
36

Med Princ Pract 2020;29:32-38 DOI: $10.1159 / 000501896$
Charfi et al. 
DRB $1{ }^{*} 01$ allele was confirmed by a meta-analysis in which serological groups DR1 and DR7 were negatively associated with pSS [9]. To better explore the HLA region and its involvement in susceptibility to pSS, we completed our HLA/pSS association study by analyzing microsatellite markers spanning the HLA region and a haplotype association study was conducted using the Haplo.stats software.

Indeed, a strongly significant positive association was found with the D6S265 marker $(p=0.00056)$. This association was independent of other HLA markers. This argues in favor of the presence of a localized susceptibility gene in the HLA class I region. In our South Tunisian population, this marker contributes to the susceptibility to other AID, such as pemphigus [24] and RA. In Caucasians, this marker has been associated with RA [25], SLE [26], and juvenile idiopathic arthritis [27]. Linkage disequilibrium with candidate genes could explain this association. Indeed, several genes located in the vicinity of this marker could be involved in the etiopathogenesis of AID: MICD, HCG9, HLA-J, ZNRD1, PPP1R11, TRIM31, and RNF39 (http://www.ncbi.nlm.nih.gov/). The ZNRD1 gene that codes for a protein involved in the regulation of cell proliferation could be a candidate gene for pSS. Hong et al. [28] have shown that the hyperexpression of this gene could be responsible for an increase in the expression of the bcl 2 protein generating a lack of apoptosis. High expression of the bcl 2 antigen has been reported in self-reactive lymphocytes accumulated in the salivary glands during pSS.

In our study, the only marker of the HLA class III region positively associated with pSS is C1_2_C (a20 allele). This association was independent of the other associated markers. Linkage disequilibrium with a candidate gene could explain this association. This region hosts four functional candidate genes (IkBL, ATP6G, BAT1, and MICB) which can be in linkage disequilibrium with the C1_2_C marker. Of these 4 genes, IkBL could be a susceptibility gene. This gene encodes a protein belonging to the NF-kB family of transcription factors and can, therefore, be involved in the regulation of the expression of pro-inflammatory cytokines such as TNFa and IL-1. Castiblanco and Anaya [29] reported an association of a polymorphism of the IkBL gene with pSS and SLE. The polymorphism of the SNP IkBL $(+738 \mathrm{C})$ is three times more likely to develop pSS $(p=0.009)$ and twice more likely to develop SLE $(p=0.01)$ compared with controls.

Several studies have suggested a direct role of TNFa in the pathogenesis of SS. TNFa favors the expression of SSA and SSB antigen on the cell surface, independently of apoptosis. The association of TNF $\alpha$ polymorphism with pSS or with one of these clinical manifestations has been reported by Guggenbuhl et al. [30]. They suggested a role for the TNFa10 allele in susceptibility to pSS. It seems to be related to joint manifestations and the presence of anti-SSA Ab [30].

In our study, no association was found with TNFa10. The TNFa2 allele was more frequent in patients compared to controls, but this was statistically insignificant. This is consistent with the results of another Tunisian study [5]. However, the TNFa6 allele has been associated in our patients with the positivity of anti-SSA Ab. The TNFa6 allele could be associated with hyperexpression of TNFa. Indeed, in humans, a pathogenic relationship has been found between some TNF microsatellite alleles (TNFa) and the amount of TNF produced.

\section{Conclusion}

This study confirms the association of the HLADRB1*03 allele with pSS in the South Tunisian population. For the HLA class I region, we have described for the first time a strong association between HLA-B15 antigen and pSS in our population. The analysis of microsatellite markers showed a strongly significant positive association with the D6S265 marker located in the HLA class I region. These results indicate the involvement of the HLA class I region in susceptibility to pSS in our South Tunisian population. This effect seems independent of the role played by the HLA-DRB1 locus. Family studies and SNP analysis within HLA class I and class III candidate genes should help clarify the role of these genes in the physiopathology of the disease.

\section{Statement of Ethics}

All patients and controls gave written informed consent for their participation in the study. This study was approved by our local ethics committee.

\section{Disclosure Statement}

The authors have no conflicts of interest to declare. 


\section{References}

1 Kassan SS, Moutsopoulos HM. Clinical manifestations and early diagnosis of Sjögren syndrome. Arch Intern Med. 2004 Jun;164(12): 1275-84.

2 Vitali C, Bombardieri S, Jonsson R, Moutsopoulos HM, Alexander EL, Carsons SE, et al.; European Study Group on Classification Criteria for Sjögren's Syndrome. Classification criteria for Sjögren's syndrome: a revised version of the European criteria proposed by the American-European Consensus Group. Ann Rheum Dis. 2002 Jun;61(6):554-8.

3 Burbelo PD, Ambatipudi K, Alevizos I. Genome-wide association studies in Sjögren's syndrome: what do the genes tell us about disease pathogenesis? Autoimmun Rev. 2014 Jul; 13(7):756-61.

4 Lessard CJ, Li H, Adrianto I, Ice JA, Rasmussen A, Grundahl KM, et al.; UK Primary Sjögren's Syndrome Registry. Variants at multiple loci implicated in both innate and adaptive immune responses are associated with Sjögren's syndrome. Nat Genet. 2013 Nov;45(11):1284-92.

5 Hadj Kacem H, Kaddour N, Adyel FZ, Bahloul Z, Ayadi H. HLA-DQB1 CAR1/ CAR2, TNFa IR2/IR4 and CTLA-4 polymorphisms in Tunisian patients with rheumatoid arthritis and Sjögren's syndrome. Rheumatology (Oxford). 2001 Dec;40(12):1370-4.

6 Mahfoudh N, Ayadi I, Kamoun A, Ammar R, Mallek B, Maalej L, et al. Analysis of HLA-A, -B, -C, -DR, -DQ polymorphisms in the South Tunisian population and a comparison with other populations. Ann Hum Biol. 2013 Jan; 40(1):41-7.

7 Gourraud PA, Mano S, Barnetche T, Carrington $\mathrm{M}$, Inoko $\mathrm{H}$, Cambon-Thomsen $\mathrm{A}$. Integration of microsatellite characteristics in the MHC region: a literature and sequence based analysis. Tissue Antigens. 2004 Nov; 64(5):543-55.

8 Marzouk S, Akrout R, Kaddour N, Frigui M, Zayen A, Bahloul Z. Syndrome de GougerotSjögren primitif : à propos de 105 cas. Rev Med Interne. 2008;29:S354.

9 Cruz-Tapias P, Rojas-Villarraga A, MaierMoore S, Anaya JM. HLA and Sjögren's syndrome susceptibility. A meta-analysis of worldwide studies. Autoimmun Rev. 2012 Feb;11(4):281-7.

10 Fye KH, Terasaki PI, Michalski JP, Daniels TE, Opelz G, Talal N. Relationshipp of HLADw3 and HLA-B8 to Sjögren's syndrome. Arthritis Rheum. 1978 Apr;21(3):337-42.
11 Morling N, Andersen V, Fugger L, Georgsen J, Halberg P, Oxholm P, et al. Immunogenetics of rheumatoid arthritis and primary Sjögren's syndrome: DNA polymorphism of HLA class II genes. Dis Markers. 1991 SepOct;9(5):289-96.

12 Loiseau P, Lepage V, Djelal F, Busson M, Tamouza R, Raffoux C, et al. HLA class I and class II are both associated with the genetic predisposition to primary Sjögren syndrome. Hum Immunol. 2001 Jul;62(7):72531.

13 Londono J, Santos AM, Peña P, Calvo E, Espinosa LR, Reveille JD, et al. Analysis of HLAB15 and HLA-B27 in spondyloarthritis with peripheral and axial clinical patterns. BMJ Open. 2015 Nov;5(11):e009092.

14 Siala M, Mahfoudh N, Fourati H, Gdoura R, Younes M, Kammoun A, et al. MHC class I and class II genes in Tunisian patients with reactive and undifferentiated arthritis. Clin Exp Rheumatol. 2009 Mar-Apr;27(2):208-13.

15 Bennani N, Atouf O, Benseffaj N, Brick C, Essakalli M. [HLA polymorphism and Behçet's disease in Moroccan population]. Pathol Biol. 2009 Jul;57(5):403-9. French.

16 Stein JM, Machulla HK, Deschner J, Fickl S, Jockel-Schneider Y, Tamm M, et al. Prevalence of periodontitis in individuals with human leukocyte antigens (HLA) A9, B15, A2, and B5. Clin Oral Investig. 2016;20(4):70310.

17 Kivity S, Arango MT, Ehrenfeld M, Tehori O, Shoenfeld Y, Anaya JM, et al. Infection and autoimmunity in Sjogren's syndrome: a clinical study and comprehensive review. J Autoimmun. 2014 Jun;51:17-22.

18 Rickinson AB, Moss DJ. Human cytotoxic T lymphocyte responses to Epstein-Barr virus infection. Annu Rev Immunol. 1997;15(1): 405-31.

19 Whittingham S, McNeilage J, Mackay IR. Primary Sjögren's syndrome after infectious mononucleosis. Ann Intern Med. 1985 Apr; 102(4):490-3.

20 Gottenberg JE, Busson M, Loiseau P, CohenSolal J, Lepage V, Charron D, et al. In primary Sjögren's syndrome, HLA class II is associated exclusively with autoantibody production and spreading of the autoimmune response. Arthritis Rheum. 2003 Aug;48(8):2240-5.

21 Anaya JM, Mantilla RD, Correa PA. Immunogenetics of primary Sjögren's syndrome in Colombians. Semin Arthritis Rheum. 2005 Apr;34(5):735-43.
22 Huang R, Yin J, Chen Y, Deng F, Chen J, Gao $\mathrm{X}$, et al. The amino acid variation within the binding pocket 7 and 9 of HLA-DRB1 molecules are associated with primary Sjögren's syndrome. J Autoimmun. 2015 Feb;57:53-9.

23 Kovács A, Endreffy E, Petri I, Kovács L, Pokorny G. HLA class II allele polymorphism in Hungarian patients with primary Sjögren's syndrome. Scand J Rheumatol. 2006 Jan-Feb; 35(1):75-6.

24 Abida O, Mahfoudh N, Kammoun A, Gaddour L, Hakim F, Toumi A, et al. Polymorphisms of HLA microsatellite marker in Tunisian pemphigus foliaceus. Hum Immunol. 2013 Jan;74(1):104-9.

25 Barnetche T, Constantin A, Gourraud PA, Abbal M, Garnier JG, Cantagrel A, et al. Microsatellite typing of the human leucocyte antigen region: analytical approach and contribution to rheumatoid arthritis immunogenetic studies. Tissue Antigens. 2006 Nov; 68(5):390-8.

26 Martens HA, Nolte IM, van der Steege G, Schipper M, Kallenberg CG, Te Meerman GJ, et al. An extensive screen of the HLA region reveals an independent association of HLA class I and class II with susceptibility for systemic lupus erythematosus. Scand J Rheumatol. 2009;38(4):256-62.

27 Smerdel A, Lie BA, Ploski R, Koeleman BP, Førre $\varnothing$, Thorsby E, et al. A gene in the telomeric HLA complex distinct from HLA-A is involved in predisposition to juvenile idiopathic arthritis. Arthritis Rheum. 2002 Jun; 46(6):1614-9.

28 Hong L, Piao Y, Han Y, Wang J, Zhang X, Du $\mathrm{Y}$, et al. Zinc ribbon domain-containing 1 (ZNRD1) mediates multidrug resistance of leukemia cells through regulation of P-glycoprotein and Bcl-2. Mol Cancer Ther. 2005 Dec;4(12):1936-42.

29 Castiblanco J, Anaya JM. The IkappaBL gene polymorphism influences risk of acquiring systemic lupus erythematosus and Sjögren's syndrome. Hum Immunol. 2008 Jan;69(1): 45-51.

30 Guggenbuhl P, Veillard É, Quelvennec E, Jego $P$, Semana G, Jean S, et al. Analyse des microsatellites a du TNF- $\alpha$ chez 35 syndromes de Gougerot-Sjögren primitifs. Rev Rhum. 2000; 67(5):362-7. 